\title{
Microbial Profile and Antimicrobial Susceptibility Pattern of Pus Culture Isolates from a Teaching Tertiary Care Hospital, South India
}

\author{
M. Subha $^{1 *}$ and Meerah Srinivasagam ${ }^{2}$ \\ ${ }^{1}$ Department of Microbiology, Madras Medical College, Chennai, Tamil Nadu, India \\ ${ }^{2}$ Department of Microbiology, Chengalpattu Govt. Medical College, \\ Chengalpattu, Tamil Nadu, India \\ *Corresponding author
}

\section{A B S T R A C T}

Pyogenic infections are one of the most common clinical complications following surgery and trauma. Prompt antimicrobial therapy is needed to reduce the morbidity of these

Keywords

Pyogenic infections, Antimicrobial susceptibility pattern, Pus samples

Article Info

Accepted:

10 March 2018

Available Online:

10 April 2018 infections. Rationale use of antibiotics is the need of the hour to curb the rising antimicrobial resistance. A retrospective study was undertaken to identify the microbial profile with their antibiogram among the pus culture isolates in a teaching tertiary care hospital from January 2017 to June 2017.504 pus culture samples processed at the microbiology lab were analyzed. The isolates from positive pus culture were identified by standard protocols and antimicrobial susceptibility patterns were identified by CLSI guidelines. Positive pus cultures were obtained in $59.92 \%(302 / 504)$. Gram positive bacteria accounted for $31.9 \%$ (97), Gram negative bacteria $67.12 \%$ (204) and Candida spp $0.99 \%$ (3) with predominance of $S$. aureus $26.32 \%$ (80) followed by E. coli $13.82 \%$ (42) and $K$. pneumoniae $13.49 \%$ (41). $17.5 \%$ of $S$. aureus was MRSA and $100 \%$ sensitive to vancomycin. $23.61 \%$ of $E$. coli and $25 \%$ of $K$. pneumoniae were ESBL producers. Majority of Gram negative isolates were sensitive to imipenem and meropenem. The present study provides useful information on the microbial profile and their antimicrobial susceptibility pattern of the pus culture isolates that may help in the formulation of antibiotic policy for pyogenic infections.

\section{Introduction}

Pyogenic infections are a significant group of infections encountered by clinicians worldwide. These infections are caused by pathogens, exogenously or endogenously, during or after trauma, burns and surgical procedures (Dryden et al., 2010). Since these infections are associated with high morbidity prompt antibiotic therapy is mandatory. An increase incidence of multidrug resistant Gram negative bacteria and Methicillin resistant Staphylococcus aureus is frequently isolated from pyogenic infections under hospital settings (Muluye et al., 2014; Trojan et al., 2016). The emergence of antibiotic resistant pathogens poses a grave threat to the health care system. The evidence based knowledge of the microbial spectrum of pyogenic infections and their antibiogram plays a crucial role in treatment and infection control practices. Henceforth this study was designed 
to analyze the pattern of pathogens and their antimicrobial susceptibility from pus culture reports in a tertiary care hospital.

\section{Materials and Methods}

A retrospective study was conducted in a tertiary care hospital in South India for a period of 6 months from January 2017 to June 2017. A total of 504 pus culture samples collected by sterile swabs and sterile syringe aspiration from clinically suspected cases of pyogenic infections were processed by standard microbiological method and antimicrobial susceptibility testing by KirbyBauer disc diffusion method as per CLSI guidelines.

A data on sociodemographic variables such gender, wards, pus culture results and their antimicrobial susceptibility pattern were collected manually from laboratory register and analyzed by counts and percentage using MS Excel,2007 version.

\section{Results and Discussion}

Out of 504 pus culture samples, 302 (59.92\%) gave positive result. From 302 culture positive samples, 171 (56.62\%) were males and 131 (43.38\%) were females yielding a male: female ratio of 1.30. Among the department wise distribution of positive pus culture isolates, Surgery showed maximum culture positivity $173(57.28 \%)$ followed by Orthopaedics 31 (10.26\%) and Plastic Surgery ward 29 (9.60\%) (Table 1). Of the 302 positive pus culture isolates, 297 yielded monomicrobial bacterial isolates, 2 yielded polymicrobial bacterial isolates and 3 yielded fungal isolates. So a total of 304 organisms were isolated. Among 301 bacterial isolates, Gram positive and Gram negative bacteria constituted 97 (31.91\%) and 204 (67.12\%) respectively.
The predominant bacterial isolate was $S$. aureus 80 (26.32\%), followed by E. coli 42 (13.82\%), K. pneumonia 41 (13.49\%), K. oxytoca 38 (12.5\%), Pseudomonas aeroginosa 31 (10.20\%), Proteus vulgaris 26 (8.55\%), Citrobacter spp 14 (4.61\%), Proteus mirabilis 10 (3.29\%), CONS 10 (3.29\%), Enterococcus spp $7(2.30 \%)$ and Acinetobacter spp 2 $(0.66 \%)$. The 3 fungal isolates were Candida non albicans spp $(0.99 \%)$ (Fig. 1).

All Gram positive isolates showed 100\% sensitivity to vancomycin. MRSA was found in $14(17.5 \%)$ and MR CONS in $2(20 \%) . S$. aureus showed a high resistance to penicillin $(56.25 \%)$, ciprofloxacin $(36.25 \%)$ and erythromycin $(31.25 \%)$ (Table 2$)$.

All Gram negative isolates were highly sensitive to imipenem and meropenem. Only $61(35.26 \%)$ of Gram negative isolates showed ampicillin sensitivity. Among Enterobacteriaceae (171/301), 88 (51.46\%) isolates were resistant to $3^{\text {rd }}$ generation cephalosporins. ESBL production in $E$. coli was 17 (23.61\%), K. pneumoniae 18 (25\%) and $K$. oxytoca 17 (23.61\%).

Enterobacteriaceae isolates showed a sensitivity pattern of $132(71.2 \%)$ to amikacin, $75(43.86 \%)$ to gentamycin, $68(39.77 \%)$ to ciprofloxacin and 40(23.4\%) to doxycycline (Table 3). Among nonfermenters, $P$. aeroginosa showed moderate sensitivity to ceftazidime $(67.74 \%)$, amikacin $(58.06 \%)$, ciprofloxacin $(51.61 \%)$ and gentamycin (48.39\%).

In this study, the pus culture positivity rate in clinically suspected cases was $59.92 \%$ which is comparable to the previous reports of Rai et al., (2017) (59\%) and Trojan et al., (2016) $(60.1 \%)$. The male: female distribution of pus isolates was found to be 1.30:1 while the study of Rao et al., 2014 showed 1.43:1 ratio. 
Fig.1 Distribution of microbial isolates among the positive pus culture $(n=304)$

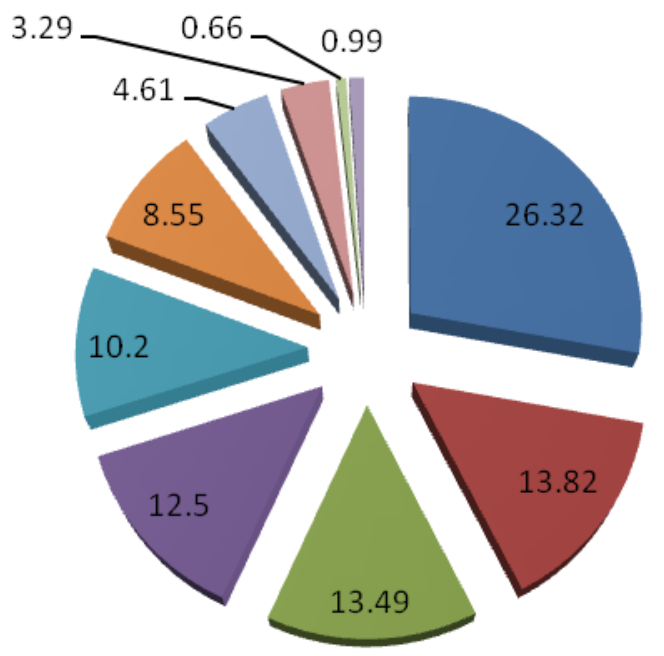

$$
\begin{aligned}
& \text { S.aureus } \\
& \text { E.coli } \\
& \text { K.pneumoniae } \\
& \text { - K.oxytoca } \\
& \text { - P.aeroginosa } \\
& \text { P.vulgaris } \\
& \text { - Citrobacter spp } \\
& \text { P.mirabilis } \\
& \text { Acinetobacter spp } \\
& \text { Candida spp }
\end{aligned}
$$

Table.1 Ward wise distribution of pus culture isolates $(n=302)$

\begin{tabular}{|l|c|c|}
\hline S. No & Ward & Number \\
\hline 1. & Surgery & $173(57.28 \%)$ \\
\hline 2. & Orthopaedics & $31(10.26 \%)$ \\
\hline 3. & Plastic Surgery & $29(9.60 \%)$ \\
\hline 4. & Medicine & $26(8.61 \%)$ \\
\hline 5. & OG & $22(7.28 \%)$ \\
\hline 6. & Skin & $21(6.95 \%)$ \\
\hline & Total & $302(100 \%)$ \\
\hline
\end{tabular}

Table.2 Antimicrobial susceptibility pattern of the Gram positive isolates in pus culture ( $\mathrm{n}=97)$
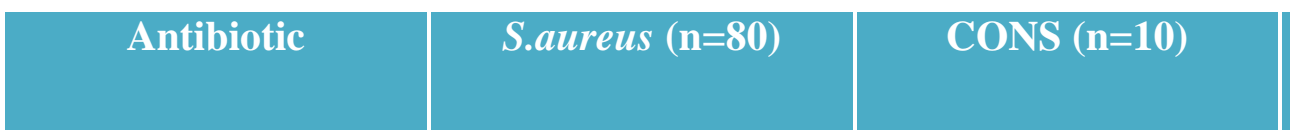

\section{Enterococcus spp

$$
(n=7)
$$

\begin{tabular}{|l|}
\hline Penicillin (10 units) \\
\hline Erythromycin $(15 \mu \mathrm{g})$ \\
\hline Cefoxitin $(30 \mu \mathrm{g})$ \\
\hline Ciprofloxacin $(5 \mu \mathrm{g})$ \\
\hline Gentamycin $(10 \mu \mathrm{g})$ \\
\hline Gentamycin $(120 \mu \mathrm{g})$ \\
\hline Vancomycin $(30 \mu \mathrm{g})$ \\
\hline
\end{tabular}

\begin{tabular}{|c|c|c|}
\hline $35(43.75 \%)$ & $6(60 \%)$ & $1(14.29 \%)$ \\
\hline $55(68.75 \%)$ & $9(90 \%)$ & $1(14.29 \%)$ \\
\hline $66(82.5 \%)$ & $8(80 \%)$ & NT \\
\hline $51(63.75 \%)$ & $8(80 \%)$ & $3(42.86 \%)$ \\
\hline $60(75 \%)$ & $9(90 \%)$ & NT \\
\hline NT & NT & $5(71.43 \%)$ \\
\hline $80(100 \%)$ & $10(100 \%)$ & $7(100 \%)$ \\
\hline
\end{tabular}

NT- not tested 
The department wise distribution revealed the dominance of Surgical department (57.28\%) which correlates well with the study conducted by Bhaskar Das et al., (2018). Majority of the pus samples yielded monomicrobial growth similar to Basista et al., (2017). Gram positive and Gram negative bacteria constituted $31.91 \%$ and $67.12 \%$ respectively. It conforms to the findings of Mantravadi et al., (2015).

In the present study S. aureus $(26.32 \%)$ was the most frequently isolated bacteria followed by E. coli $(13.82 \%)$ and $K$. pneumoniae $(13.49 \%)$ which is in agreement with the study of Mantravadi et al., (2015) that reported S. aureus $(37.2 \%)$, E. coli $(21.7 \%)$ and Klebsiella spp (16.8\%).

Other studies showed different distribution of bacterial isolates which may be attributed to the differences in study design, geographical location and climate conditions.

In the present study $14(17.5 \%)$ of S. aureus was MRSA similar to Chauhan et al., (2015). All the isolates of $S$. aureus were sensitive to vancomycin as comparable with Sujatha et al., (2016). The ESBL producing Enterobacteriaceae in our study was $42.10 \%$ of which E. coli was $23.61 \%$ and $K$. pneumoniae $25 \%$ in contrast to the study by Shrestha et al., (2011) where the prevalence of ESBL was $18 \%$ of which $E$. coli was $53.7 \%$ and $K$. pneumoniae $14.8 \%$.

This increase of ESBL producing Enterobacteriaceae in our study is a matter of concern. The Gram negative isolates also showed an increasing resistance to ampicillin, gentamycin, fluoroquinolones and $3^{\text {rd }}$ generation cephalosporins similar to that of the findings of Javeed et al., (2011). Similarly high sensitivity to carbapenems of Gram negative isolates corroborates with the findings of Vijeta et al., (2015).
In the clinical care pyogenic infections are still a leading cause of morbidity following surgery and trauma. Emergence of multidrug resistant pathogens poses a challenge in treating these infections as there is limited availability of newer antimicrobial agents in the pipeline. Since the microbial profile and antimicrobial susceptibility pattern may change from time to time and place to place, this study will guide the clinicians in proper selection of antibiotics for successful treatment and also for formulating hospital antibiotic policy.

\section{References}

Basista Prasad Rijal, Deepa Satyal, and Narayan Prasad Parajuli. 2017. High Burden of Antimicrobial Resistance among Bacteria Causing Pyogenic Wound Infections at a Tertiary Care Hospital in Kathmandu, Nepal. Journal of Pathogens, Article ID 9458218, 7 pages.

Bhasar Das, Deepti Adhana, Neha Rathod et al., 2018. Aerobic Bacterial Profile and Antimicrobial susceptibility pattern in pyogenic infections in a tertiary care hospital in North India. International Journal of Science and Research; 7(2):451-454.

Chauhan M, Manish S and S. Mahajan. 2015. Aerobic Bacterial Profile and antibiotic sensitivity pattern of pus isolates in a tertiary care Hospital. Int.J.Curr.Microbiol.App.Sci; 4(5): 784787.

CLSI. Performance standard for Antimicrobial susceptibility testing. 2016; twenty third informational supplement. M100-S26: vol 33 no.1.

Dryden, M.S., 2010. Complicated skin and soft tissue infection. Journal of Antimicrobial Chemotherapy; 65(3): pp. iii35-iii44. 
Javeed I, Hafeez R, Anwar MS. 2011. Antibiotic susceptibility pattern of bacterial isolates from patients admitted to a tertiary care hospital in Lahore. Biomedica; 27: 19-23.

Mantravadi H.B, Chinthaparthi M. R, Shravani V. 2015. Aerobic isolates in pus and their antibiotic sensitivity pattern: a study conducted in a teaching hospital in Andhra Pradesh. International Journal of Medical Science and Public Health; 4(8): 107679.

Muluye, D., Y. Wondimeneh, G. Ferede et al., 2014. Bacterial isolates and their antibiotic susceptibility patterns among patients with pus and/or wound discharge at Gondar university hospital. BMC Research Notes; 7(1).

Rai, S., U. N. Yadav, N. D. Pant et al., 2017. Bacteriological profile and antimicrobial susceptibility patterns of bacteria isolated from pus/wound swab samples from children attending a tertiary care hospital in Kathmandu, Nepal. International Journal of Microbiology; Article ID 2529085, 5 pages.

Rao R, Basu R, Biswas DR. 2014. Aerobic bacterial profile and antimicrobial susceptibility pattern of pus isolates in a south Indian tertiary care hospital. Journal of Dental and Medical Sciences March; 13(3): 59-62.

Shrestha, S., R. Amatya and R. Dutta. 2011. Prevalence of Extended spectrum beta lactamase production in gram negative isolates from pyogenic infection in tertiary care hospital of Eastern Nepal. Nepal Med Coll J; 13(3):186-189.

Sujatha R, Ankita Tripathi, Vineet, Nidhi Pal. 2016. Microbial Characterisation of Pus Isolates and the Changing Trends in their Sensitivity Pattern at a Tertiary Care Hospital in Kanpur City. RAMA Univ. J. Med Sci; 2(3):1-7.

Trojan, R., L. Razdan, and N. Singh. 2016. Antibiotic susceptibility patterns of bacterial isolates from pus samples in a tertiary care hospital of Punjab, India. International Journal of Microbiology; Article ID 9302692: 4 pages.

Vijeta Sharma, Geeta Parihar, Vijaylaxmi Sharma, Harshita Sharma. 2015. A Study of Various Isolates from Pus Sample with Their Antibiogram from JLN Hospital, Ajmer. IOSR Journal of Dental and Medical Sciences; 14(10): 64-68.

\section{How to cite this article:}

Subha, M. and Meerah Srinivasagam. 2018. Microbial Profile and Antimicrobial Susceptibility Pattern of Pus Culture Isolates from a Teaching Tertiary Care Hospital, South India. Int.J.Curr.Microbiol.App.Sci. 7(04): 1149-1153. doi: https://doi.org/10.20546/ijcmas.2018.704.126 